\title{
Tracing the Origin of Bars and Bulges through the Study of their Stellar and Ionised Gas Properties
}

\author{
Isabel Pérez ${ }^{1,2}$ Patricia Sánchez-Blázquez ${ }^{3}$ Almudena Zurita ${ }^{1,2}$ and \\ Estrella Florido ${ }^{1,2}$ \\ ${ }^{1}$ Dep. Física Teórica y del Cosmos, Campus de Fuentenueva, Universidad de Granada, Spain \\ ${ }^{2}$ Instituto Carlos I de Física Teórica y Computación, Spain \\ ${ }^{3}$ Dep. Física Teórica, Universidad Autonoma de Madrid, Cantoblanco, 28049, Madrid, Spain
}

\begin{abstract}
We have carried out a detailed analysis of stellar properties of bars and bulges of a sample of early-type galaxies. We have also compared the results of the bulge properties with the bulges of a similar sample of unbarred galaxies, deriving the SSP equivalent stellar parameters in the same way as for our sample. We are currently characterising the nebular gas in the bulges of the sample galaxies. We have found differences in the bulge stellar population properties between barred and unbarred galaxies. The bulges of barred galaxies seem to be more metal rich, at a given velocity dispersion $(\sigma)$, than the bulges of unbarred galaxies, as measured by some metallicity sensitive indices. There are indications that the ratio of relative abundance of alpha-elements with respect to iron, $[\mathrm{E} / \mathrm{Fe}]$, derived for the bulges of barred galaxies tend to lie above the values of the unbarred galaxies at a given $\sigma$. We also find three different types of bars according to their metallicity and age distribution along the radius: 1) Bars with negative metallicity gradients. They show mean young/intermediate population $(<2$ Gyr $)$, and have amongst the lowest stellar maximum central velocity dispersion of the sample. 2) Bars with null metallicity gradients. These galaxies that do not show any gradient in their metallicity distribution along the bar and have negative age gradients (i.e younger populations at the bar end). 3) Bars with positive metallicity gradients, i.e. more metal rich at the bar ends. These galaxies are predominantly those with higher velocity dispersion and older mean population. We find no significant correlation between the age and metallicity distribution, and bar/galaxy parameters such as the AGN presence, size or the bar strength. From the kinematics, we find that all the galaxies show a disk-like central component. Regarding the ionised gas properties, we find that the nebular metallicity gradients are shallower than the stellar ones, indicating an efficient mixing of the material currently forming. The nebular central metallicities do not show a correlation with the central $\sigma$, and, furthermore, in some cases the nebular metallicities are clearly lower than the stellar ones, indicating an external origin for the gas fueling the current star formation. All these results point to a scenario, at least for early-type galaxies, where bars are long-lived and where the formation of the bulge and the bar are closely linked. Later accretion of gas can then be funneled towards the center forming nuclear discs and rings, producing substructures with low level of star formation, but that would not contain enough stars to produce a change in the SSP-equivalent metallicities and $[\mathrm{E} / \mathrm{Fe}]$.
\end{abstract}

Keywords. Galaxies: spirals- Galaxies: abundances - Galaxies: evolution - Galaxies: formation - Galaxies: structure - Galaxies: kinematics

\section{Introduction}

We are carrying out a project to determine whether bars are long-lasting and to characterise the role played by bars in the building up of the central bulge. Stellar population parameters in the bar and the bulge region can provide with archeological' clues as to the 
formation and evolution of the bar. The project consists of obtaining stellar population parameters as derived from stellar absorption line-strength indices and to determine the properties of the ionised gas associated to the bar and bulge regions. Our final sample comprises 20 early-type barred galaxies (morphological types ranging from $\mathrm{S} 0$ to $\mathrm{Sb}$ ). For an analysis of the differences between the bulges of barred and unbarred galaxies our sample needed to be complemented with a similar sample of galaxies without bars. So, in addition to the bulges of barred galaxies, we carried a comparison with Moorthy et al. (2006) data from a sample of both, barred and unbarred galaxies, being the only sample available in the literature with similar characteristics to our data; namely, spectral coverage and resolution, morphological type and inclination values. The data have been re-analysed in the same way as for our sample. This is the first time that such study is done for a large sample of barred galaxies, with a total of 31 barred galaxies combining both samples. We refer the reader to the following papers for detailed information on the different aspects of the project; the results of the stellar bulge properties have been published in Pérez \& Sánchez-Blázquez (2011, A\&A accepted) while the results on the bar stellar population properties were presented in Pérez, Sánchez-Blázquez \& Zurita $(2007,2009)$. The work on the ionised gas is currently on-going and will be shortly submitted.

In order to relate the stellar population properties with the morphological features, we perform an isophotal analysis on available archived $R$-band images of the galaxy sample. The minimum ellipticity in the bar region has been adopted as the definition for the size of the bar. It has been shown (Michel-Dansac \& Wozniak 2006) that this measurement is correlated with the corotation radius and, therefore, gives a bar structural size, it is also the method to calculate the bar size that is the least sensitive to dust absorption. The bulge radius is considered as the radius where the $P A$ and ellipticity $\epsilon$ starts showing the typical bar profile, i.e. increasing $\epsilon$ and constant $P A$. The general morphology of the galaxies in the sample shows a large range of properties. Some galaxies, independently of their type, have outer rings, some host strong bars and some are of intermediate $A B$ type. This allows us to test systematic behaviors associated to different morphologies.

\section{Kinematics of the sample galaxies}

From the study of the kinematics along the bar, we have found that all the galaxies in the sample show a disk-like component in their centers, showing as dips or plateaus in their central stellar velocity dispersion and as stellar rotating disks. Therefore, this implies that bars, as expected, have a strong influence in the building up and later evolution of the central component.

\section{Stellar bar properties: mean values and metallicity gradients}

The mean ages of the stellar populations in our sample of bars exhibit a large dispersion. The differences in the mean ages in the bars between the different galaxies can be as large as 10 Gyr, with some galaxies showing a mean bar age larger than 10 Gyr and others younger than $<1$ Gyr. There is a trend in which galaxies with smaller central velocity dispersion are also the ones showing younger mean-ages. In the same way, galaxies with lower maximum central velocity dispersion tend to have lower values of $[\mathrm{E} / \mathrm{Fe}]$ and metallicity. We did not find a correlation neither between the mean age of the stars in the bars and the morphological type of the galaxies, although we do not have late spirals in our sample, nor with the presence of AGN. 
Galaxies with velocity dispersions higher than $170 \mathrm{kms}^{-1}$ tend to have positive metallicity gradients, while the lower velocity dispersion galaxies show a larger dispersion in their metallicity gradient value. Similar behavior is found for the age gradient, where galaxies with large velocity dispersion tend to have a shallow positive age gradient while for the lower velocity dispersion galaxies the absolute value of the age gradient is larger, both negative and positive gradients.

According to the metallicity gradient in the bar we find three types of bars:

- Bars with negative metallicity gradient (more metal poor at the ends of the bar). These bars show a mean young/intermediate population and have among the lowest velocity dispersion. They also tend to have positive age gradients, circumnuclear region $\mathrm{s}$ with young population. Since we expect the original metallicity gradient to flatten, this might be indicating that these bars formed recently.

- Bars with null metallicity gradients. These bars show gradients both in age and metallicity compatible with the models.

- Bars with positive metallicity gradients (more metal rich at the ends of the bar). These bars show older mean ages and higher velocity dispersion. Since the diffusion time-scale is short (Wozniak 2007) they are not likely to be a relic from the initial disk gradient. There is still not a conclusive explanation for the origin of this gradient yet. It is however possibly indicating an old bar.

We find no clear dependence of the gradients with the presence of AGN. We do not find neither a correlation between the mean age/metallicity of the bar with the presence of an AGN nor a correlation with the strength of the bar. There is no apparent correlation with morphological type, although we do not cover late morphological types.

\section{Stellar bulge properties: mean values and metallicity gradients}

We have found differences in the bulge stellar population properties between barred and unbarred galaxies. We find that some of the metallicity sensitive indices of the bulges of barred galaxies lie above those of unbarred galaxies and the Balmer indices tend to follow the opposite trend, as was already hinted by Moorthy et al. (2006). It is interesting to note that although one could say that there is a good correlation between line-strength indices and $\sigma$, it would be a better description of the index distribution with $\sigma$ to say that there is an almost constant value of the indices with $\sigma$ plus a tail in the distribution for the smaller $\sigma$ galaxies (below $\approx 150 \mathrm{~km} \mathrm{~s}^{-1}$ ). Most galaxies show negative gradients for metal-sensitive indices while the opposite is true for Balmer line indices, although NGC 1832 and NGC 2665 show clearly the opposite trend. The index distributions are closely linked to morphological substructures in the bulge region such as nuclear rings.

The central parts of barred galaxies tend to be more metal rich than the unbarred counterparts. Interestingly, $[\mathrm{E} / \mathrm{Fe}]$ seems to be enhanced for the barred galaxies over the unbarred central regions. Although, due perhaps to the low number of unbarred galaxies in the sample, these trends are not statistically significant and would need to be followed up with a larger sample of unbarred galaxies. The age gradients follow the index trend with $\sigma$, most galaxies show no gradient or slightly positive gradient; however, the distribution of gradients for galaxies with $\sigma$ below $\approx 150 \mathrm{~km} \mathrm{~s}^{-1}$ shows a larger dispersion. The same behaviour is found for the metallicity trends, with most values being negative, with grad $[\mathrm{Fe} / \mathrm{H}]$ around -0.05 , the only outliers are found in galaxies with $\sigma$ below $150 \mathrm{~km} \mathrm{~s}^{-1}$. Galaxies with $\sigma$ above $150 \mathrm{~km} \mathrm{~s}^{-1}$ show a null [E/Fe] gradient while galaxies with central velocity dispersion below this value show a positive $[\mathrm{E} / \mathrm{Fe}]$ gradient. We have calculated the gradients also in Moorthy's data. There is a very good agreement in the values of both samples and we do not find any difference between barred 
and unbarred galaxies. The interpretation of the gradients is difficult as there are too many processes that can modify their shape. Furthermore, the presence of substructures, as disks or rings, which are very common in our sample, makes very difficult to measure the real gradient of the spheroid. There is a good correlation between the metallicities in both the bar and the bulge, this points to a bulge enrichment linked to that of the bar.

\section{Ionised gas bulge properties}

We are currently investigating the properties of the ionised gas in the bulge region. Preliminary results show that the nebular metallicity gradients are shallower than the stellar ones, indicating an efficient mixing of the material currently forming. The nebular central metallicities do not show a correlation with the central $\sigma$, as opposed to the stellar central metallicity $\sigma$ clear relation. Furthermore, in some cases the nebular metallicities are clearly lower than the stellar ones, indicating an external origin for the gas fueling the current star formation in the bulge region.

\section{Conclusions}

The picture emerging from the results regarding the stellar populations of the bar and bulge regions and their comparison with the bulges of unbarred galaxies is that the bar formation is closely linked to the formation of the bulge. The processes can create both, the bar and the bulge, in particular in those galaxies with early type morphologies (see, e.g., Berentzen et al. 2004; Peirani et al. 2009). In this scenario, it could well happen that this mechanism created both, the bar and the bulge, in some galaxies, in particular those with early-type morphologies. During the formation of the bulge, the star formation could have been enhanced by the presence of this bar, increasing the metallicity and the $[\mathrm{E} / \mathrm{Fe}]$ values of the bulge with respect to those bulges lacking this structure (e.g. Pérez \& Freeman 2006). Later accretion of gas can then be funneled towards the center forming nuclear discs and rings (Emsellem et al. 2001; Wozniak et al. 2003, Wozniak \& Champavert 2006), producing substructures with low level of star formation, i.e. slightly younger (showing a lower SSP-equivalent age in our data), but that would not contain enough stars to produce a change in the SSP-equivalent metallicities and $[\mathrm{E} / \mathrm{Fe}]$ as the metallicity and $[\mathrm{E} / \mathrm{Fe}]$ values reflect more the values of the dominant (in mass) stellar population (see Serra \& Trager 2007), while the SSP-equivalent age are very biased towards the youngest components. In order to test this hypothesis, further work and analysis would be needed to derive star formation histories.

\section{References}

Berentzen, I., Athanassoula, E., Heller, C. H., \& Fricke, K. J. 2004, MNRAS, 347, 220

Emsellem, E., Greusard, D., Combes, F., et al. 2001, A\&A, 368, 52

Michel-Dansac, L. \& Wozniak, H. 2006, A\& $A$, 452, 97

Moorthy, B. \& Holtzman, J. 2006, MNRAS, 371, 583

Serra, P. \& Trager, S. 2007, MNRAS, 374, 769

Peirani, S., Hammer, F., Flores, H., Yang, Y., \& Athanassoula, E. 2009, A\&AA, 496, 51

Pérez, I., Sánchez-Blázquez, P., \& Zurita, A. 2007, A\&A, 465, 9

Pérez, I., Sánchez-Blázquez, P., \& Zurita, A. 2009, A\&A, 495, 775

Pérez, I. \& Freeman, K. 2006, A\&GA, 454, 165

Wozniak, H. \& Champavert, N. 2006, MNRAS, 369, 853

Wozniak, H., Combes, F., Emsellem, E., \& Friedli, D. 2003, A\&AA, 409, 469 